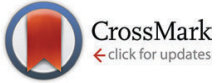

Cite this: DOI: $10.1039 / c 6 c p 06982$

\title{
Inorganically coated colloidal quantum dots in polar solvents using a microemulsion-assisted method $\dagger$
}

\author{
María Acebrón, ${ }^{\mathrm{ab}}$ Facundo C. Herrera, ${ }^{\mathrm{c}}$ Martín Mizrahi, ${ }^{\mathrm{C}}$ Cristina Navío, \\ Ramón Bernardo-Gavito, $\ddagger^{\mathrm{b}}$ Daniel Granados, ${ }^{\mathrm{b}}$ Félix G. Requejo*c and \\ Beatriz H. Juarez ${ }^{* a b}$
}

\begin{abstract}
The dielectric nature of organic ligands capping semiconductor colloidal nanocrystals (NCs) makes them incompatible with optoelectronic applications. For this reason, these ligands are regularly substituted through ligand-exchange processes by shorter (even atomic) or inorganic ones. In this work, an alternative path is proposed to obtain inorganically coated NCs. Differently to regular ligand exchange processes, the method reported here produces core-shell NCs and the removal of the original organic shell in a single step. This procedure leads to the formation of connected NCs resembling 1D worm-like networks with improved optical properties and polar solubility, in comparison with the initial CdSe NCs. The nature of the inorganic shell has been elucidated by X-ray Absorption Near Edge Structure (XANES), Extended X-ray Absorption Fine Structure (EXAFS) and X-ray Photoelectron Spectroscopy (XPS). The 1D morphology along with the lack of long insulating organic ligands and the higher solubility in polar media turns these structures very attractive for their further integration into optoelectronic devices.
\end{abstract}

Received 12th October 2016 Accepted 5th December 2016

DOI: $10.1039 / c 6 c p 06982 g$

www.rsc.org/pccp

\section{Introduction}

${ }^{a}$ Applied Physical Chemistry Department, Universidad Autónoma de Madrid, Cantoblanco, 28049, Madrid, Spain

${ }^{b}$ IMDEA Nanoscience, Faraday 9, Cantoblanco, 28049, Madrid, Spain. E-mail: beatriz.hernandez@imdea.org

${ }^{c}$ Instituto de Investigaciones Fisicoquímicas Teóricas y Aplicadas (INIFTA), CONICET and FCE, UNLP, CC/16, suc 4, 1900 La Plata, Argentina. E-mail: requejo@inifta.unlp.edu.ar

$\dagger$ Electronic supplementary information (ESI) available: Sketch of the experimental procedure followed and suggested mechanisms for transferring NCs to the water micelle (Fig. S1), TEM images of treated NCs (Fig. S2), HRTEM images of treated NCs (Fig. S3), SAXS results of samples in microemulsion (Fig. S4), fitted SAXS parameters for a homogeneous sphere of radii $r$ (Table S1), fitted SAXS parameters for a core@shell model sphere of radii of core $r_{\mathrm{c}}$ and shell thickness $t$ (Table S2), correlation effect SAXS analysis (at low $q$ values) (Fig. S5), best-fitted parameters obtained for a rigid sphere model on SAXS results are shown in Fig. S5 (Table S3), P/Cd ratios for initial CdSe cores and samples treated with TA and TC (Table S4), FTIR spectra of initial CdSe and treated NCs (Fig. S6), HRTEM images of initial CdSe NCs (Fig. S7), CdSe core characterization by XAS (Fig. S8), EXAFS fitted parameters for Se coordination shell on CdSe initial core samples in comparison with CdSe bulk reference compounds (Table S5), size histograms of initial and treated NCs (Fig. S9), EXD data (Table S6), photoluminescence response of TA + ZnAc and TC + ZnAc treated samples after one month (Fig. S10), XPS measurements of TA + ZnAc and TC + ZnAc treated samples (Fig. S11), fitted values to $\mathrm{Zn}$ K-edge EXAFS oscillations, shown in Fig. 5d, for the average coordination number $(N)$, interatomic distance $(R)$ and Debye-Waller factor $\left(\sigma^{2}\right)$ (Table S7) and K-Se and K-Zn EXAFS oscillation fittings of first coordination shell on initial and treated NCs (Fig. S12). See DOI: 10.1039/c6cp06982g

¥ Present address: Physics Department, Lancaster University, LA1 4YB, UK.
The current technology demands very efficient, robust and versatile emitting systems for their integration as active layers into optoelectronic devices. To this aim, colloidal semiconductor nanocrystals (NCs) are promising candidates due to their tunable electronic and optical properties. ${ }^{1,2}$ Although fine control over size, size-distribution, shape, and composition of colloidal NCs has been achieved, further control over the surface chemistry turns essential for their integration into devices, where radiative and non-radiative paths, electronic coupling and carrier mobilities govern their conductivity, and eventually, their performance. ${ }^{3,4}$

Since 1993, the hot-injection ${ }^{5}$ method has proved its versatility and superior potential to control size, shape, composition and crystallinity, as the NCs are produced in high boiling point solvents. By means of this method it is possible to obtain NCs with different configurations such as core/shell, dot/rod, etc., where the electron and hole wavefunctions are modulated at will. ${ }^{4}$ One example of a strongly confined system is the CdSe/ZnS core-shell NCs in which the wide band gap of ZnS compared to CdSe leads in high quantum efficiencies and photo-stability. However, the use of non-polar solvents and organic surfactants regularly yields hydrophobic NCs with a long insulating capping shell that limits their use in optoelectronic devices. Since interparticle connection and electronic coupling govern their efficiency, the regular organic insulating 
capping shell is exchanged by conductive, shorter or even atomic ligands, such as halides. ${ }^{6}$

During the last few years, the so-called inorganic ligands have attracted special attention because they provide polar soluble NCs and reduce the insulating electrical barrier of organic ligand shells favoring electrical conductivity in 2D quantumdot monolayers. Among the inorganic ligands, chalcogenides, hydrochalcogenides, mixed chalcogenides, halometallate ligands as well as hydroxy or amino functional groups have been used to replace the initial insulating shells. This replacement yields increased carrier mobilities, for both electrons and holes, and better conductivity. ${ }^{3,7-9}$ As drawbacks, in many cases, the use of efficient NCs as starting material requires multiple step reactions and post-treatment procedures are needed to obtain the ligand exchanged NCs. Also, the use of smelly or toxic reactants and the need for a strict oxygen-free working environment may limit their usage.

In this work we report on an alternative approach to grow inorganic shells on CdSe NCs, which implies the removal of the original long insulating ligands to a large extent simultaneously with the fabrication of core-shell structures under friendly working conditions (room temperature and ambient atmosphere). The procedure reported here yields coated colloidal NCs with better optical properties than the initial ones and increased solubility in polar solvents such as ethanol, isopropanol or formamide. The employed treatment combines the Successive Ionic Layer Adsorption Reaction (SILAR) and the use of Water-in-Oil (W/O) microemulsions as reaction media to grow controlled monolayers on CdSe seeds previously produced by hot-injection. In these $\mathrm{W} / \mathrm{O}$ microemulsions the small drops of water act as nanoreactors in which the shell growth takes place.

The use of microemulsions for NC synthesis dates from the 1980's, when Brus and coworkers were able to produce CdSe NCs with tunable ligand spheres. ${ }^{10}$ During the following decade a great effort was made to improve this methodology to synthesize $\mathrm{CdS}$, CdSe, and $\mathrm{Cd}_{y} \mathrm{Zn}_{1-y} \mathrm{~S}$ NCs with different morphologies, ${ }^{11}$ CdS superlattices, ${ }^{12}$ and $\mathrm{CdS} @ \mathrm{SiO}_{2}$ nanocomposites. ${ }^{13}$ There are certain benefits derived from the use of microemulsions as nanoreactors, such as the performance of lower rate reactions and ligand exchange procedures without precipitation at room temperature (RT) (for a given composition), in well suspended NC dispersions. Examples of concomitant ligand removal and shell formation have been previously reported in the coverage of oleic acid, dodecylamine and octadecylamine capped-NCs with $\mathrm{SiO}_{2},{ }^{14}$ which renders the final encapsulated nanocomposites soluble in solvents of higher polarity.

The combination of these methodologies, namely, hot injection, SILAR, and microemulsions, involves the removal of the initial ligand shell to a large extent and triggers the connection between NCs. In contrast to regular ligand exchange processes, where the NCs are regularly synthesized by multiple-step reactions and the insulating ligands are replaced to provide NC compatibility, the here proposed procedure benefits from the lack of organic ligands and, at the same time, allows the synthesis of type I or quasi-type II core-shell structures. These new NCs form 1D networks with increased solubility in polar media and improved optical properties. We believe these features can be very promising to obtain NCs for further progress in the formation of NC solids with expected high carrier mobilities or active layers in optoelectronic devices.

\section{Experimental}

\section{Chemicals}

Cadmium oxide Puratronic (CdO, 99.998\%) and octadecylphosphonic acid (ODPA, 97\%) were purchased from Alfa Aesar. Trioctylphosphine (TOP, 90\%), Igepal CO-520 (poly(5)oxyethylene4-nonylphenylether), thioacetamide (TA, $\geq 99.0 \%$ ), thiocarbamide (TC, $\geq 99.0 \%$ ), zinc acetate dihydrated (ZnAc, $\geq 98.0 \%$ ), octadecene (ODE, 90\%) and Coumarin 6 (98\%) were purchased from Sigma Aldrich. Trioctylphosphine oxide (TOPO, 98\%) was purchased from Merck. Absolute ethanol (99.8\%), cyclohexane (99.5\%), formamide ( $\geq 99.5 \%$ ), isopropanol ( $\geq 99.8 \%$ ) and chloroform (99.6\%) were purchased from Sharlau. ZnS, hydrated $\mathrm{ZnSO}_{4}, \mathrm{CdSO}_{4}$ and $\mathrm{ZnO}$ were used as references for X-ray Absorption Near Edge Structure (XANES) spectroscopy. All chemicals were used directly without further purification.

\section{CdSe NC synthesis}

The CdSe NCs were synthesized according to a previously reported hot-injection method with some modifications. ${ }^{15}$ For the reaction, $0.06 \mathrm{~g}$ of $\mathrm{CdO}, 3 \mathrm{~g}$ of TOPO and $0.280 \mathrm{~g}$ of ODPA were placed in a three neck round flask under an inert atmosphere and the temperature was set at $150{ }^{\circ} \mathrm{C}$. At this temperature a red solution was obtained and degassed for one hour under vacuum. Later, the temperature was increased to $340{ }^{\circ} \mathrm{C}$ for Cd (ODPA) complex formation, leading to a colorless solution. Once the complex is formed, the temperature was decreased to $300{ }^{\circ} \mathrm{C}$ and $1.8 \mathrm{ml}$ of TOP was added. Further, the temperature was increased to $320{ }^{\circ} \mathrm{C}$ and $0.43 \mathrm{ml}$ of a Se@TOP solution ( $0.058 \mathrm{~g}$ of Se dissolved in $0.360 \mathrm{~g}$ of TOP) was injected. Afterwards, the heating mantel was removed and the solution was left to cool to room temperature (RT). Once the NCs are formed the solution was filtered through a $0.45 \mu \mathrm{m}$ PTFE filter to remove the unreacted organic products and the sample was washed by four cycles of centrifugation and re-dispersion using hexane as a solvent and methanol as a non-solvent.

\section{Shell synthesis in microemulsion}

Once the ODPA capped NCs were synthesized, they were transferred to a W/O microemulsion containing $0.62 \mathrm{~g}$ of Igepal CO-520 as a surfactant, $100 \mu \mathrm{l}$ of water and $12 \mathrm{ml}$ of cyclohexane. For each microemulsion the water/igepal/cyclohexane ratio was set to 4.9/0.8/94.3 (v/v). The S and $\mathrm{Zn}$ precursors were also added as microemulsions with similar compositions where the salts were previously dissolved in the water used for microemulsion preparation. For the preparation of these precursor microemulsions a maximum amount of salts soluble in $100 \mu \mathrm{l}$ of water were dissolved and the necessary quantity of surfactants and cyclohexane needed to maintain the water/igepal/cyclohexane ratio was added to these salt solutions. 
The concentration and particle size of the initial NCs are estimated from both TEM and UV-Vis spectra, ${ }^{16}$ and the necessary amount of $\mathrm{S}$ and $\mathrm{Zn}$ precursors for the formation of one monolayer of $\mathrm{ZnS}$ was calculated according to the SILAR method. ${ }^{17}$ The necessary amount of $\mathrm{S}$ microemulsion was calculated taking into the account the $\mathrm{S}$ precursor concentrations and was added to the NC microemulsion under stirring (500 rpm) and the mixture was stirred for 48 hours at RT. Later, the calculated amount of $\mathrm{Zn}$ microemulsion was added and stirred for further 48 hours. Once the NCs were produced, they were washed by centrifugation and re-dispersion cycles ( 5 cycles) with chloroform and ethanol. Finally, they were dissolved in polar solvents (formamide, ethanol and isopropanol). The main disadvantage of using microemulsions is the exhaustive cleaning, necessary to isolate the NCs from the microemulsion medium. This is usually overcome by the use of high volumes of solvent/non-solvent during the washing procedure.

\section{Characterization}

\section{Steady-state spectroscopy}

Optical absorption measurements were carried out using a Varian spectrophotometer (Cary 50). Photoluminescence (PL) spectra were recorded on a spectrofluorometer (Horiba Jobin Yvon Fluoromax-4) using an excitation wavelength of $420 \mathrm{~nm}$ and acquiring corrected spectra. The samples were placed in a quartz cuvette and the concentration was maintained constant for comparison of the initial and treated NCs.

\section{Quantum yield measurements}

The relative Quantum Yield (QY) was measured by comparison with an emitting standard (Coumarin 6). ${ }^{18}$ The QYs were calculated in different media and the refractive indexes of the solvents were estimated using a hand-held refractometer model 330. The concentrations were fixed below 0.1 optical density to avoid reabsorption effects.

\section{Time resolved spectroscopy}

The decay rates were acquired employing a time correlated single photon counting card (PicoQuant, TimeHarp 260 PICO Single) with 25 ps base resolution. The samples in solution were placed in a quartz cuvette and pumped with a tunable supercontinuum laser (Fianium SC400+ superchrome) delivering $10 \mathrm{ps}$ long pulses with $\lambda=450 \mathrm{~nm}$ and a repetition rate of $40 \mathrm{MHz}$ (25 ns). The emission is collected with a multi-core optical fiber and passed through a $0.5 \mathrm{~m}$ spectrometer (Andor Shamrock) with a $300 \mathrm{l} \mathrm{mm}^{-1}$ grating. The filtered emission was detected using an avalanche photodiode (TAU-SPAD-250) with $<250$ dark counts per second, a timing resolution of $300 \mathrm{ps}$ and a detection efficiency of $50 \%$ at $550 \mathrm{~nm}$. The measured instrument response function is $600 \mathrm{ps}$ at $550 \mathrm{~nm}$.

\section{Transmission electron microscopy (TEM)}

Low resolution Transmission Electron Microscopy (TEM) images were obtained using a JEOL JEM 1010 microscope operating at $100 \mathrm{kV}$. High Resolution Electron Transmission Microscopy (HRTEM) images and Energy Dispersive X-Ray (EDX) analysis were obtained using a JEOL 2100F microscope operating at $200 \mathrm{kV}$ and equipped with an EDX detector INCA $\mathrm{x}$-sight from Oxford Instruments. Size histograms were obtained from HRTEM images. HRTEM images at low acceleration voltages were obtained at Centro Nacional de Microscopía Electrónica at Universidad Complutense de Madrid on a JEOL JEM ARM200cF microscope operated at $80 \mathrm{kV}$ working in Transmission and Scanning-Transmission (HRSTEM) mode in bright and dark fields and using a High-Angle Annular Dark-Field (HAADF) detector to acquire the elemental mappings. The elemental distribution was performed using Electron Energy Loss Spectroscopy (EELS).

\section{X-ray photoelectron spectroscopy (XPS)}

XPS measurements were performed under Ultra High Vacuum (UHV) conditions (with a base pressure of $7 \times 10^{-10} \mathrm{mbar}$ ) using a monochromatic Al K-alpha line as the exciting photon source $(h \nu=1486.7 \mathrm{eV})$, a hemispherical energy analyzer (SPHERA-U7, analyzer pass energy was set to $20 \mathrm{eV}$ for the XPS measurements to have a resolution of $0.6 \mathrm{eV}$ ) and to compensate the built up charge on the sample surface during the measurements the use of a Flood Gun (FG-500, Specs) with low energy electrons of $3 \mathrm{eV}$ and $40 \mu \mathrm{A}$ was necessary.

\section{Small angle X-ray scattering (SAXS)}

SAXS experiments were performed at INIFTA (La Plata, Argentina) facilities using a XEUSS 1.0 equipment from XENOCS with a $\mathrm{K} \alpha$-Copper radiation microsource. A PILATUS-100K detector was used with a sample detector distance of $513 \mathrm{~mm}$. Onedimensional curves were obtained by integration of the $2 \mathrm{D}$ data using the Foxtrot program. The scattering intensity distributions as a function of the scattering vector $(q)$ were obtained in the $q$ range between 0.028 and $0.65 \AA^{-1}$. The samples were placed in borosilicate glass capillary tubes of $1.5 \mathrm{~mm}$ diameter and $10 \mu \mathrm{m}$ wall thickness.

\section{X-ray absorption (XAS)}

XAS experiments were measured at the SXS (S and Cd edges) and XAFS2 ( $\mathrm{Zn}$ and Se edges) beamlines at the LNLS (Laboratorio Nacional de Luz Sincrotron), Campinas, Brazil. X-ray Absorption Near Edge Structure (XANES) spectroscopy measurements at the S K (2472 eV) and Cd L3 (3538 eV) edges were carried out using a double-crystal monochromator equipped with $\mathrm{InSb}(111)$ crystals giving an energy resolution of $2 \mathrm{eV}$ at the $\mathrm{S}$ K-edge and $1 \mathrm{eV}$ at the Cd L3-edge. Experiments were performed in a vacuum of $10^{-9}$ mbar at RT. The incident beam intensity (I0) was measured using a thin foil of $\mathrm{Al}$ located before the main chamber. Samples were dropped on carbon tape to be measured in Total Electron Yield (TEY) and fluorescence modes, collecting the emitted current for each incident photon-energy with an electrometer connected to the sample. To avoid self-absorption effects ${ }^{19}$ only results for TEY mode are presented and analyzed in this work. The photon energies were calibrated using a Mo or Ag metallic foil and setting the first inflection point to the energy of the 
Mo L3 absorption edge $(2520 \mathrm{eV})$ for $\mathrm{S}$, and to the $\mathrm{Ag} \mathrm{L} 2$ absorption edge $(3524 \mathrm{eV})$ for $\mathrm{Cd}$ measurements. The final XANES spectra were obtained after background subtraction and normalization to the postedge intensity. Extended X-ray Absorption Fine Structure (EXAFS) experiments at the $\mathrm{Zn} \mathrm{K}$ edge $(9659 \mathrm{eV})$ spectra were measured at room temperature using a $\mathrm{Si}(111)$ single channel-cut crystal monochromator in fluorescence mode. An ionization chamber was used to detect the incident flux and a 15-element germanium solid-state detector was used to sense the fluorescence signal from the sample. $\mathrm{ZnSO}_{4}$, $\mathrm{ZnS}$ and ZnAc reference samples were measured in transmission mode with two ion chambers as detectors. The EXAFS data were extracted from the measured absorption spectra by standard methods using the ATHENA software which is part of the IFFEFIT package. $^{20}$ The Fourier transforms were calculated using the Hanning filtering function. EXAFS modeling was carried out using the ARTEMIS program (IFFEFIT package). Structural parameters (coordination numbers, bond lengths and their Debye-Waller factor) were obtained by a nonlinear least-squares fit of the theoretical EXAFS signal to the data in $R$ space by Fourier transformation of both the experimental and calculated data. Theoretical scattering path amplitudes and phase shifts for all paths used in the fits were calculated using the FEFF code. ${ }^{21}$ The passive reduction factor $\mathrm{S} 02$ was restrained to the value of 0.86. This value was obtained fitting the EXAFS spectrum of metallic $\mathrm{Zn}$ foil constraining the coordination number of the first coordination shell to 12 .

\section{Fourier transform infrared spectroscopy (FTIR)}

FTIR spectra were acquired using a FT-IR spectrum 100 Perkin Elmer spectrometer equipped with an Attenuated Total Reflectance (ATR) module. The samples were prepared by drop-casting and dried using air flow. OPDA powders and cyclohexane were measured directly.

\section{Results and discussion}

Fig. 1 shows the procedure followed where ODPA-capped CdSe NCs, synthesized according to a previously reported hot-injection method with some modifications, ${ }^{15}$ are dispersed in a W/O microemulsion by addition of Igepal, water and cyclohexane to the CdSe NC dispersion (from (a) to (b)). The shell formation by SILAR is triggered by the controlled addition of the $S$ precursor first (c) and, secondly, the $\mathrm{Zn}$ precursor (d). The precursor solutions are equally prepared in W/O microemulsions (see the Experimental section for details). The coating reaction and ligand removal may take place in these water drops while after washing and redispersion in polar solvents (e) the particle connection occurs. Further details about the experimental procedure can be found in Fig. S1, ESI. $\dagger$

In this work, two different sulfur precursors were used, namely, thioacetamide (TA) and thiocarbamide (TC) which, despite their structural similarities, yielded NCs with different optical properties and thickness coverages. For the Zn-containing microemulsion medium zinc acetate $(\mathrm{ZnAc})$ was chosen based

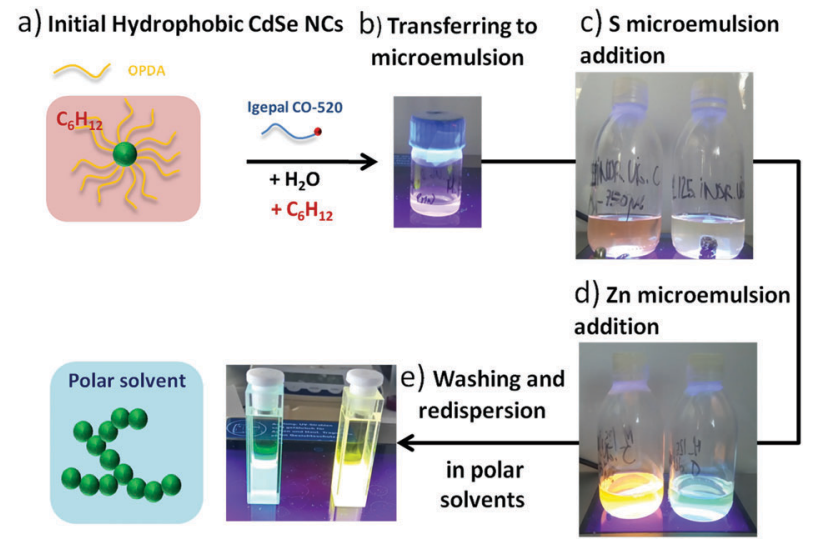

Fig. 1 Sketch of the methodology that combines SILAR with water-in-oil microemulsions. CdSe NCs produced by hot injection (a) are included in a microemulsion (b), where different S (c) and Zn precursors (d) are added. Later the samples were washed and redispersed in polar solvents (e).

on its previously reported reactivity with $\mathrm{S}^{2-}$ to obtain $\mathrm{ZnS}$-coated NCs. $^{22}$ These precursors, with mild reactivity, have been widely used to grow CdS and ZnS thin films by chemical bath deposition and CdS NCs. ${ }^{23}$

Fig. 2 shows TEM, STEM and HRTEM images of initial and treated NCs. After the treatment in microemulsion, the initial CdSe NCs (Fig. 2a) yielded connected NCs in worm-like 1D structures (Fig. $2 \mathrm{~b}$ and c). These networks are obtained regardless of the use of TA + ZnAc or TC + ZnAc (more images can be found in Fig. S2 and S3, ESI $\dagger$ ). As evidenced by the HRTEM images (Fig. 2c and Fig. S3, ESI $\dagger$ ), the NCs are not fused following the same crystallization orientation (no evidence of oriented attachment is observed), ${ }^{24}$ but organized in a randomly oriented network. We ascertained that these 1D structures are formed during washing, as no aggregation is visible by SAXS experiments performed with NCs in microemulsion (Fig. S4, S5 and Tables S1-S3, ESI $\dagger$ ). In the case reported here, the formation of these $1 \mathrm{D}$ structures is mainly promoted by the lack of initial ODPA ligands which are removed to a large extent after TA or TC treatments, although interparticle dipole-dipole interactions may also contribute. ${ }^{15,25}$ Indeed, the connected network points to a lack of organic ligands between NCs. This ligand removal is ascertained by EDX (Table S4, ESI $\dagger$ ), showing that the initial $\mathrm{P}$ content (from phosphonic species) decreases $70 \%$ after the coverage treatments. Also, the observed differences in the FTIR spectra of initial and treated NCs support the removal of phosphonic species (Fig. S6, ESI $\dagger$ ).

The CdSe NC size distribution obtained from HRTEM images (Fig. S7, ESI $\dagger$ ) is $2.6 \pm 0.2 \mathrm{~nm}$ according to statistics obtained from at least $300 \mathrm{NCs}$, and in good agreement with the $2.5 \mathrm{~nm}$ diameter estimated from the UV-Vis spectrum ${ }^{16}$ and data extracted by XANES spectroscopy and EXAFS experiments at the Se K-edge (Fig. S8 and Table S5, ESI $\dagger$ ). The average diameters of the NC networks upon treatment obtained by HRTEM are $3.7 \pm 0.4 \mathrm{~nm}$ for TA + ZnAc NCs and $2.7 \pm 0.3 \mathrm{~nm}$ for TC $+\mathrm{ZnAc}$ ones, evidencing a thicker shell for TA + ZnAc treatment. Size histograms of initial and treated NCs can be found in Fig. S9, ESI. $\dagger$ 


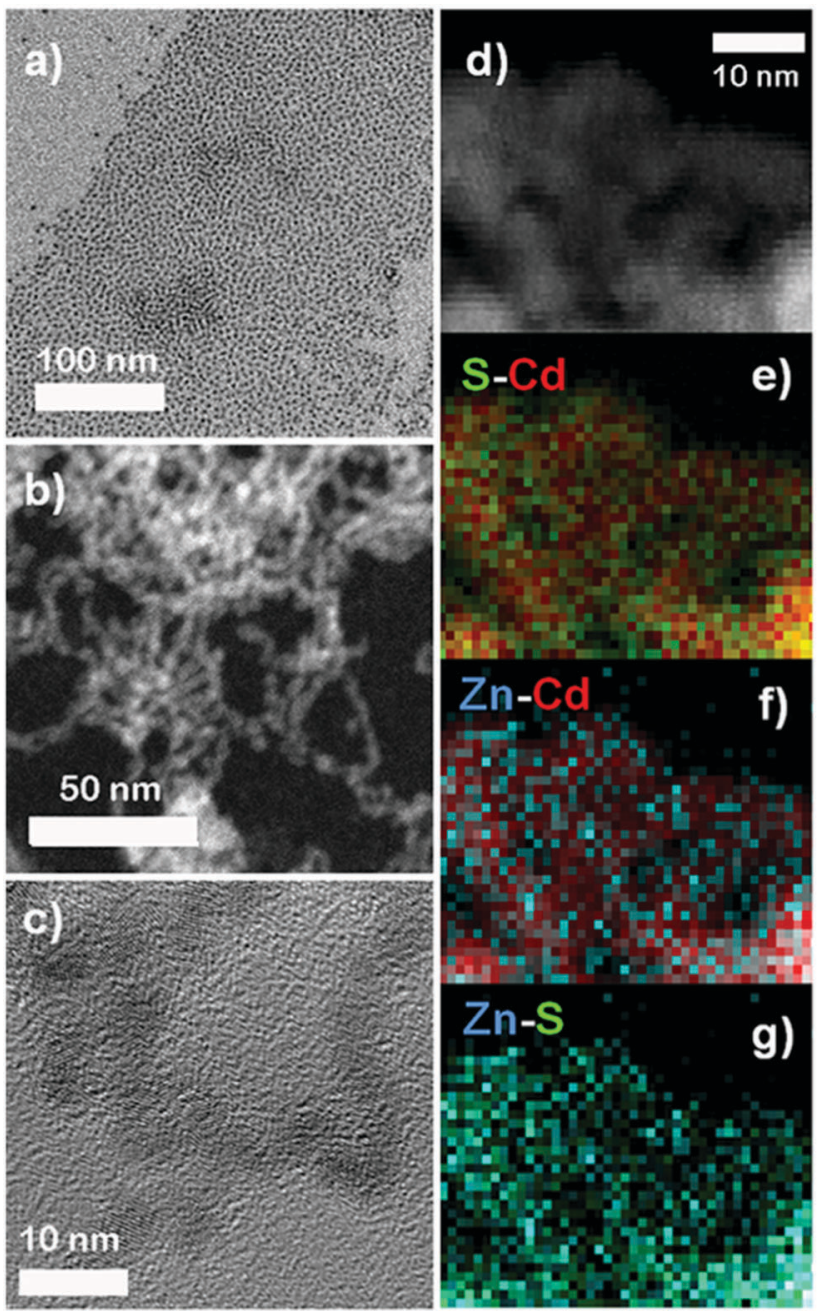

Fig. 2 (a) TEM image of initial CdSe NCs. (b) and (c) Correspond to a STEM and HRTEM images, respectively, of the $1 \mathrm{D}$ networks obtained after the coating process. Lack of original ligands is inferred in the NC networks, where the well-defined distances between NCs in the original colloidal dispersions are removed. (e-g) Show EELS elemental analysis maps of $\mathrm{TA}+\mathrm{ZnAc}$ treated NCs acquired at $80 \mathrm{kV}$ of the region shown in (d) (scale bar $10 \mathrm{~nm}$ ) evidencing the presence of $\mathrm{Zn}$ and $\mathrm{S}$ as coating.

Indeed, the formation of a thicker shell for TA + ZnAc treated NCs is also evidenced by SAXS, where the data were fitted, after evaluation of different geometries, sizes and distributions, to a core@shell model of homogeneous and rigid spheres (Fig. S4 and Tables S1, S2, ESI $\dagger$ ), being this geometry the compatible one with not only SAXS but also EXAFS experimental results. We attribute the thicker film formation to the higher decomposition rate of TA compared to TC for $\mathrm{S}^{2-}$ production, as previously reported. ${ }^{26}$ Elemental distribution profiles for TA + ZnAc treated samples were obtained by EELS (Fig. 2d-g) working in the STEM mode and using the HAADF detector. The maps and STEM images are recorded at $80 \mathrm{kV}$ in order to preserve the stability of the samples under the irradiation beam. The elemental maps in Fig. 2e and f, corresponding to the area selected in Fig. 2d, show a layer of S and $\mathrm{Zn}$ around the underneath NCs. Filtering the cores, a homogenous Zn and $\mathrm{S}$ layer through the whole network is evidenced in Fig. $2 \mathrm{~g}$.

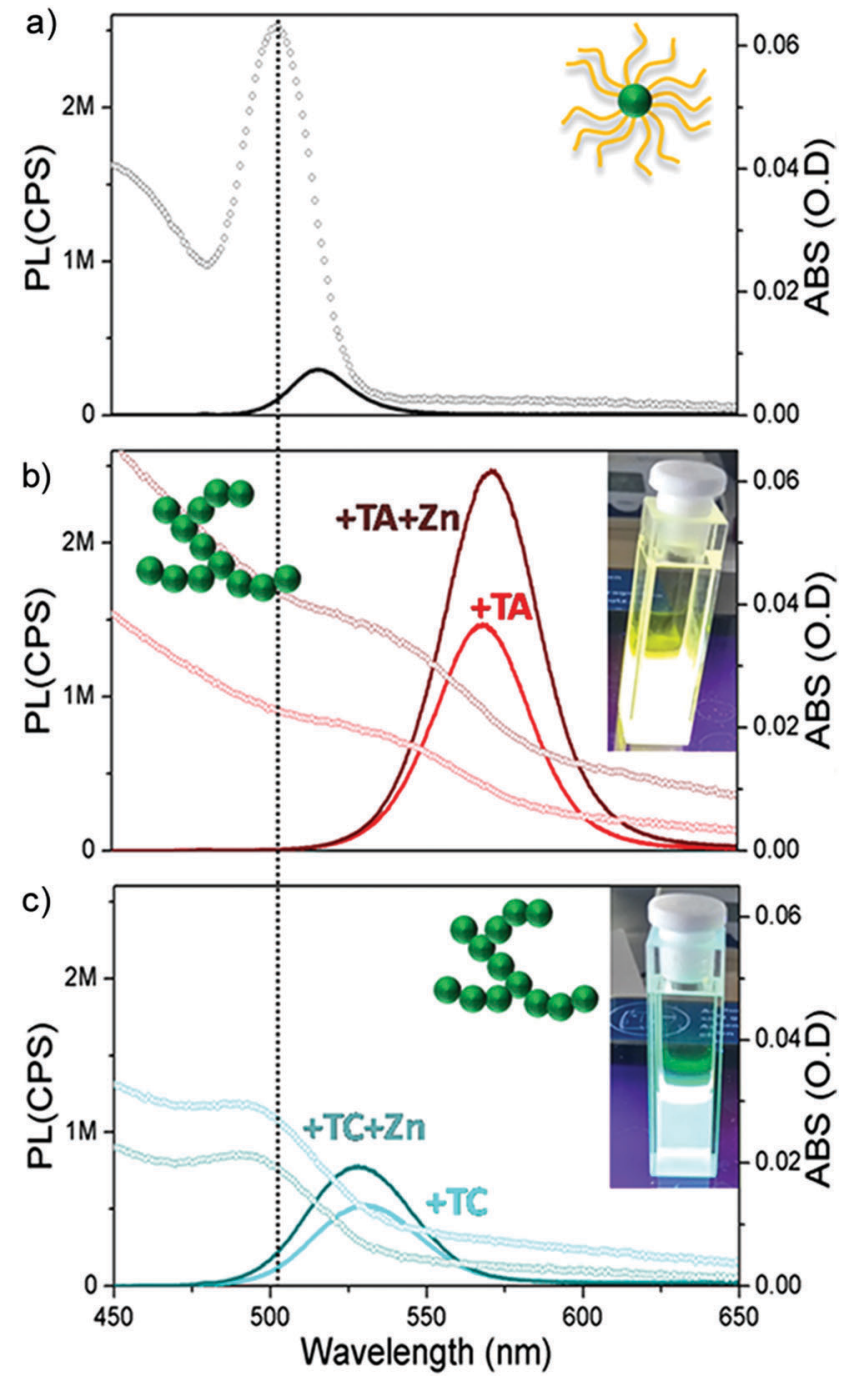

Fig. 3 Absorption (open circles) and emission spectra (full lines) of initial NCs (a), NCs treated with TA and TA + ZnAc (b) and NCs treated with $\mathrm{TC}$ and $\mathrm{TC}+\mathrm{ZnAc}$ (c). All spectra were acquired in the microemulsion medium. Insets show network dispersions in isopropanol.

Fig. 3 shows the optical properties of initial CdSe NCs (Fig. 3a), TA + ZnAc (Fig. 3b) or TC + ZnAc treated NCs (Fig. 3c). For TA treated NCs (Fig. 3b), a redshift in the optical properties compared to the initial cores is apparent during the first addition of TA (red line) and a further slight shift upon addition of the $\mathrm{Zn}$ precursor (brown line). The observed total redshift of the absorption edge is $44 \pm 10 \mathrm{~nm}$. This shift can be explained assuming well-known type-I or quasi type-II core-shell $\mathrm{NCs},{ }^{1}$ where a concomitant increase in the photoluminescence (PL) response is also recorded. In contrast, in the case of TC + ZnAc treated NCs (Fig. 3c), a blueshifted absorption edge $(-13 \pm 5 \mathrm{~nm})$ and only initial moderate PL increments (slightly red-shifted respect to the initial cores) are observed. These values are calculated for at least 10 different samples for each treatment.

As evidenced by EDX (Table S6, ESI $\dagger$ ), for TA and TA + ZnAc treated samples the $\mathrm{S}$ content is higher than for TC and $\mathrm{TC}+\mathrm{ZnAc}$ treated ones (for comparable reaction times), which 
would explain the previously mentioned formation of a thicker shell and a more red-shifted optical response, as reported in Fig. 3b compared to Fig. 3c.

On the other hand, the blueshifted absorption edge for TC treated samples could be explained by partial surface oxidation and concomitant mean effective size reduction of the cores. ${ }^{27,28}$ However, since no evidence of oxidized Cd species is detected by XANES (further discussed in Fig. 6), the degradation of the CdSe cores can be discarded. Likewise, since the blue shifted absorption edge is evident before $\mathrm{Zn}$ addition, $\mathrm{Zn}_{x} \mathrm{Cd}_{1-x} \mathrm{Se}$ alloyed structures can also be discarded. ${ }^{27,29}$ Other factors affecting the absorption edge include the substitution of long aliphatic ligands by shorter and more electronegative ones, ${ }^{30}$ and/or a slight reduction of the particle size produced by a feasible moderate etching of the CdSe cores by TC. In addition, the treated samples show improved stability in polar solvents, as is observed in the optical images shown as insets in Fig. 3b and c corresponding to treated samples in isopropanol.

In order to characterize in more detail the effects of the coverage on the optical properties of the CdSe cores, the decay dynamics of initial and treated NCs are analyzed and summarized in Fig. 4 and Table 1. Fig. 4 a and Table 1 show the decay dynamics of initial CdSe NCs (1), NCs treated with TA (2) and $\mathrm{TA}+\mathrm{ZnAc}$ in microemulsion (3), and in ethanol (4). The same data for NCs treated with TC (5) and TC + ZnAc ( 6 and 7) can be found in Fig. $4 \mathrm{~b}$ and Table 1 . The decay rates of both initial and treated NCs fit quite well with a double exponential function suggesting that multiple processes are involved in the decays. From these fittings two different decay rates can be calculated: $\tau_{1}$, corresponding to a very fast decay which has been previously related to non-radiative processes involving surface defects, and
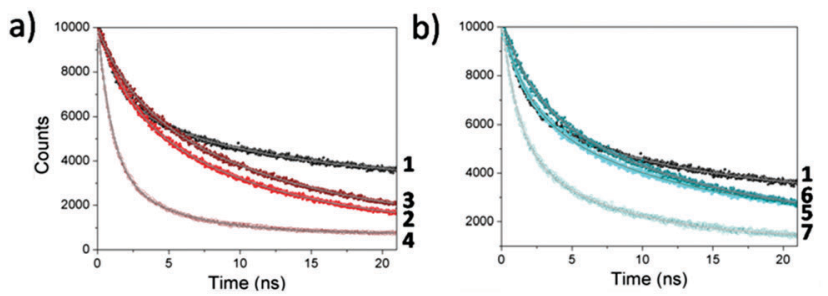

Fig. 4 (a) Decay dynamics (dotted lines) and double exponential fittings (solid lines) of initial NCs (black (1)), TA treated NCs (red (2)), TA + ZnAc treated NCs (wine (3)) and washed TA + ZnAc treated NCs redispersed in ethanol (empty wine dots (4)). (b) Decay dynamics of initial NCs (black (1)), TC treated NCs (cyan(5)), TC + ZnAc treated NCs (dark cyan (6)) and washed $\mathrm{TC}+\mathrm{ZnAc}$ treated NCs redispersed in ethanol (empty dark cyan dots (7)).

Table 1 Lifetime values and fitting parameters of initial and treated NCs measured in microemulsion and ethanol

\begin{tabular}{llrl}
\hline Sample & $\tau_{1}(\mathrm{~ns})$ & $\tau_{2}(\mathrm{~ns})$ & $R$ \\
\hline (1) Initial NCs (microemulsion) & 1.5 & 11.3 & 0.99718 \\
(2) NCs + TA (microemulsion) & 1.9 & 9.9 & 0.99892 \\
(3) NCs + TA + ZnAc (microemulsion) & 2.2 & 11.3 & 0.99877 \\
(4) NCs + TA + ZnAc (EtOH) & 1.0 & 4.8 & 0.99908 \\
(5) NCs + TC (microemulsion) & 1.8 & 11.3 & 0.99838 \\
(6) NCs + TC + ZnAc (microemulsion) & 2.0 & 12.1 & 0.99834 \\
(7) NCs + TC + ZnAc (EtOH) & 1.2 & 7.1 & 0.99793
\end{tabular}

$\tau_{2}$, which has been ascribed to the excitonic recombination. ${ }^{31}$ While the faster decay rate increases slightly for both treated samples with respect to the initial CdSe NCs in microemulsion, it decreases slightly in ethanol pointing to a possible passivation effect of the surfactant (IGEPAL), or TA-Cd(Zn) and TC-Cd(Zn) complexes anchored to the formed shell, according to FTIR spectra (Fig. S6, ESI $\dagger$ ). ${ }^{32}$ Although visual inspection of Fig. 4 could suggest that treated NCs in microemulsion decay faster than the initial ones, this is the consequence of two effects: (1) the major contribution of $\tau_{1}$ to the decay curves of the treated samples, probably related to inhomogeneous surface passivation and the plausible NC interactions, and (2) an instrumental response limitation, given by the repetition rate used $(40 \mathrm{MHz})$ which produces the mixing of the end of the slower decays with the start of a new excitation cycle. In fact, the slower decay rate, $\tau_{2}$, remains practically constant for any treatment in microemulsion and only decreases in ethanol, which can be the result of either the already mentioned removal of the ligands or complexes during the washing procedure or the interparticle connection triggering electronic coupling in the 1D structures, as previously observed for chalcogenide NCs, ${ }^{9}$ or even both of them.

Fig. 5 shows the temporal evolution of the QYs as the precursors are added to both types of samples in a microemulsion, and the final QYs measured in isopropanol as the polar medium. Maximum QY values of $10 \%$ and $20 \%$ in alcohol are obtained for samples treated with TC + ZnAc (Fig. 5a) and TA + ZnAc (Fig. 5b), respectively. This means that using an experimentally comfortable method (RT and ambient conditions) we are able to produce polar soluble NCs free of insulating ligands to a large extent presenting, at least, 4 times higher QYs than the original hydrophobic ones. The QY values for TA + ZnAc treated CdSe cores in isopropanol are comparable to QYs obtained for NCs coated with inorganic ligands. ${ }^{8}$ Interestingly, while the QY values in microemulsion

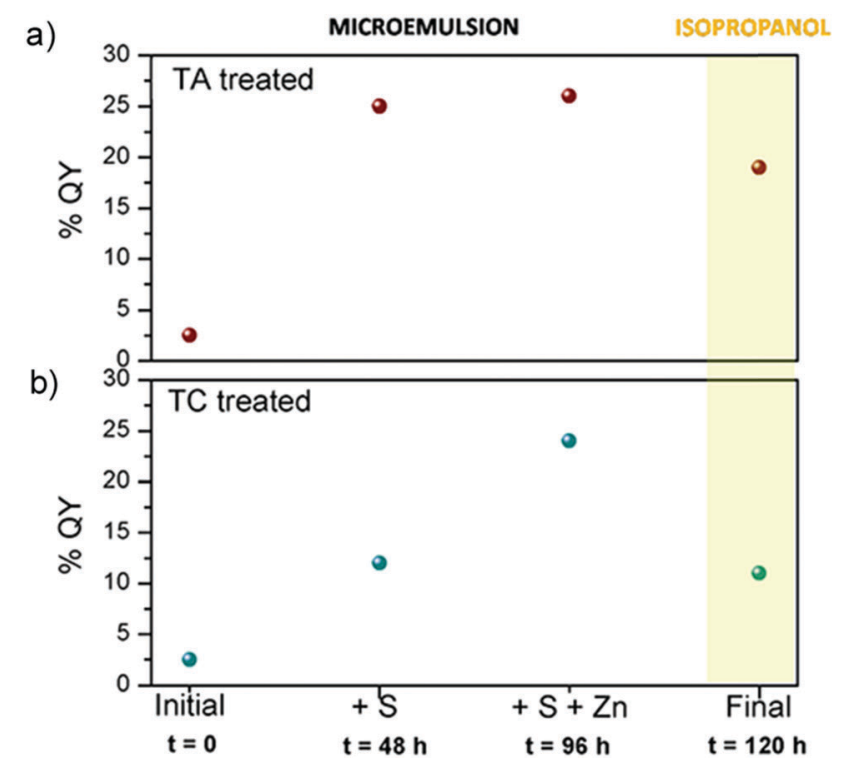

Fig. 5 QYs of TA and TA + ZnAc treated NCs (a) and TC and TC + ZnAc treated NCs (b) in microemulsion. Final QYs after treatment and the washing procedure measured in isopropanol. 
are initially larger for TA treated samples, the opposite tendency is observed for TC treated ones. These last samples show increased PL response for longer reaction times (after 1 month), indicating a possible surface reconstruction with time according to the slower decomposition rate of TC (Fig. S10, ESI $\dagger$ ). ${ }^{26}$

In order to understand the nature of the grown shells and the NC composition, synchrotron XAS measurements were performed. XANES results can be seen in Fig. 6 where Fig. $6 \mathrm{a}$ includes the S-K edge measurements for the samples treated with TA + ZnAc and TC + ZnAc. The spectra of hydrated $\mathrm{ZnSO}_{4}$ and $\mathrm{CdSO}_{4}$ are also included for comparison. As can be seen in both samples, two main peaks at 2474 and $2483 \mathrm{eV}$ can be observed. The former peak is present as a major contribution in sulfide compounds, and the latter is present in the sulfate salts. ${ }^{19,33}$ Thus, according to the S-K edge spectra a combination of sulfides and sulfates seems feasible, although out of these spectra, clear identification to $\mathrm{ZnS}$ and/or CdS cannot be ascribed univocally and the contribution of $\mathrm{Cd}$ or $\mathrm{Zn}$ sulfides and sulfates cannot be distinguishable from these results. The presence of sulfides and sulfates in both samples is also ascertained by XPS (Fig. S11, ESI $\dagger$ ) where signals close to $162 \mathrm{eV}$ are assigned to sulfides and the more oxidized signals close to $170 \mathrm{eV}$ could be associated with sulfates. The additional Cd L-XANES (Fig. 6b) and Zn K-XANES (Fig. 6c) results allow a direct interpretation about sulfide and sulfate species. a)

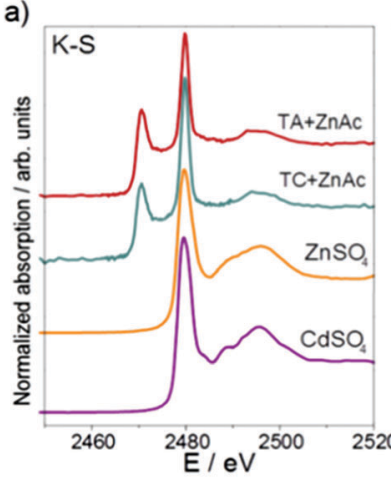

c)

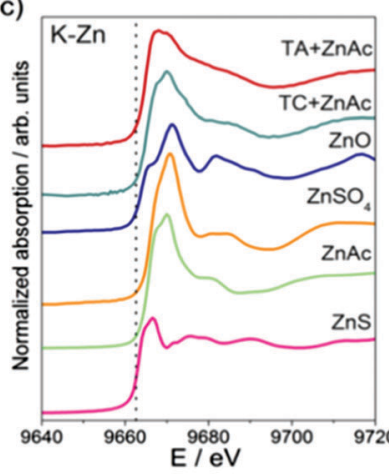

b)

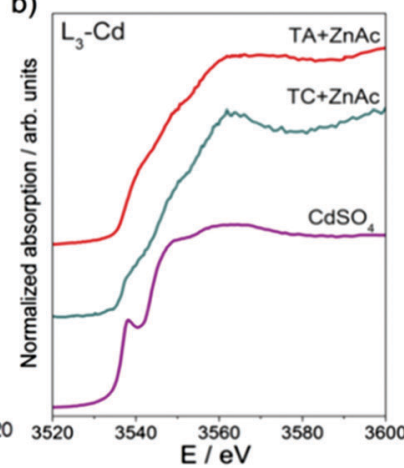

d)

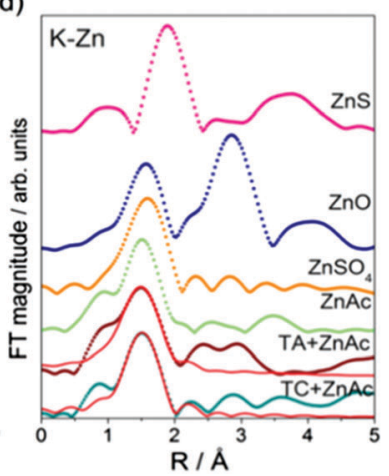

Fig. 6 XANES characterization of TA $+\mathrm{ZnAc}$ and TC $+\mathrm{ZnAc}$ treated samples along with reference compounds at the K S-edge (a), L3 Cd-edge and $\mathrm{Zn} \mathrm{K}$-edge (b), K Zn-edge (c). Vertical dotted line in (c) indicates the energy position for the Zn K-edge. Fourier transform of Zn K-edge EXAFS oscillations of the samples and reference $Z n$-compounds (d). Red full lines correspond to fitted functions (see Table S7, ESI $\dagger$ ).
The Cd XANES spectra of TA + ZnAc and TC + ZnAc samples drastically differ from $\mathrm{CdSO}_{4}$ (Fig. 6b), whose Cd L-XANES spectrum shows a pre-peak at $3538 \mathrm{eV}$. Moreover, both Cd XANES spectra corresponding to our samples are very close to that already reported for CdS. ${ }^{34}$

However, as reported in Table S6, ESI, $\dagger$ the initial Se content decreases for both TA and TC treated samples concomitantly to the increase of $\mathrm{S}$ content, which is probably related to the formation of a $\mathrm{CdSe}_{1-x} \mathrm{~S}_{x}$ layer. In both treated samples the $\mathrm{S}+\mathrm{Se} / \mathrm{Cd}$ ratio remains constant after sulfur addition, suggesting that an intermediate $\mathrm{CdSe}_{1-x} \mathrm{~S}_{x}$ shell is also rather feasible. In this sense, our Cd L-XANES characterization is also compatible with the presence of $\mathrm{CdSe}_{1-x} \mathrm{~S}_{x}$, already reported elsewhere. ${ }^{35}$

Additionally, from Zn K-XANES (Fig. 6c) and Zn K-edge EXAFS (Fig. 6d) results, the chemical state of $\mathrm{Zn}$ in our samples with respect to several reference compounds can be compared. In both cases the observations are conclusive with respect to the presence of sulfide and sulfate species. According to the energy edge position (see the vertical reference line in Fig. 6c) and general features of the spectra, the chemical state of $\mathrm{Zn}$ in both NCs cannot be associated with $\mathrm{ZnS}$ and is closer to oxidized $\mathrm{Zn}$-species, in particular close to $\mathrm{ZnSO}_{4}$, which is in principle reasonable since this is the outer element in the NCs. This is confirmed by EXAFS results just by the only inspection of the first coordination shell from the Fourier transform of EXAFS oscillations for each sample and reference compound (Fig. 6d and Table S7, ESI $\dagger$ ). It is important to note that XANES and EXAFS experiments at the Zn K-edge were performed in microemulsion and samples were not exposed to air. This means that any change at the chemical state of $\mathrm{Zn}$ is only due to the chemistry of the synthesis. In contrast, XANES experiments at the Cd L-edge were performed in a vacuum after dropping the liquid sample on carbon tape. Since the chemical Cd-state is not oxidized this implies the good stability of the core of the NCs. The fittings of all EXAFS oscillations performed on initial cores and TA + ZnAc and TC + ZnAC samples can be found in Fig. S12, ESI $\dagger$.

Gathering the whole picture, the closest description of the final NC composition is compatible with a CdSe core with a $\mathrm{CdSe}_{1-x} \mathrm{~S}_{x}$ and/or CdS intermediate shell, which is responsible for the optical behavior of the samples according to type I or quasi type II structures and a more external oxidized shell of Zn-species, as suggested by the sketches depicted in Fig. 7.

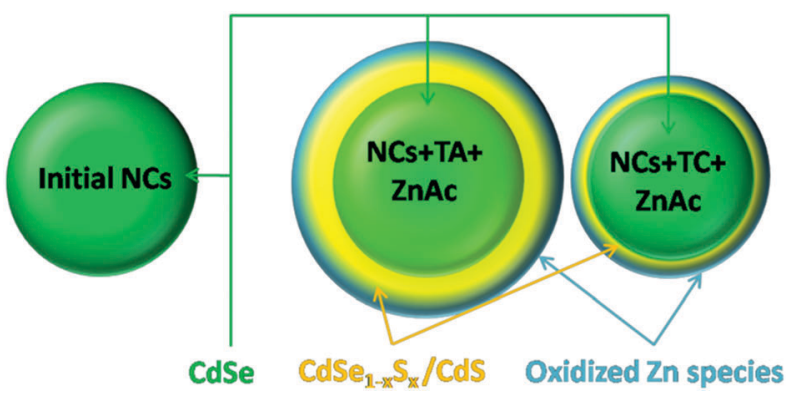

Fig. 7 Suggested morphology for initial NCs and NCs treated with $\mathrm{TA}+\mathrm{ZnAc}$ and $\mathrm{TC}+\mathrm{ZnAc}$, respectively. For the sake of clarity the coverage thickness has been depicted as homogeneous, although the formation of deposits with non-uniform thickness is feasible. 
The treatments yield 1D NCs with improved optical properties relative to the initial CdSe cores. All samples show improved stability in formamide, isopropanol and ethanol with respect to non-polar solvents such as toluene or cyclohexane. While the solvent coordination to metal surface atoms and solvent density may help to stabilize the worm-like NCs, the presence of the more oxidized external shell of undefined $\mathrm{Zn}$ species could be responsible for the increased stability in polar media. Furthermore, the presence of TA-Cd(Zn) and TC-Cd(Zn) complexes detected by FTIR can also contribute to the surface charge stabilization of the $1 \mathrm{D}$ nanostructures.

\section{Conclusions}

In summary, a combined methodology including the Successive Ionic Layer Adsorption Reaction (SILAR) and water-in-oil (W/O) microemulsions is applied to control the growth of inorganic layers on previously produced CdSe seeds. The procedure involves almost the complete elimination of the original phosphonate ligand shell simultaneously to the synthesis of core-shell structures, and yields 1D worm-like NC structures. The obtained NCs are composed of several species, including sulfides and more oxidized ones, as confirmed by X-ray Absorption Near Edge Structure, Extended X-ray Absorption Fine Structure and X-ray Photoelectron Spectroscopy. The developed procedure improves the optical properties of the initial NCs according to type-I or quasi type-II core-shell structures, reduces their solubility in nonpolar media and increases the sample stability in polar media such as isopropanol, ethanol, and formamide. These networks showing higher solubility in polar media can be advantageous to produce active layers for optoelectronic applications.

\section{Acknowledgements}

$\mathrm{BHJ}$ and MA thank for funding in the frame of the following projects: S2013/MIT-2740 from Comunidad de Madrid, MAT2013-47395-C4-3-R and FIS2015-67367-C2-1-P from the Spanish Ministry of Economy and Competitiveness. $\mathrm{BHJ}$, FCH, MM ad FGR thank CEAL-AL/2015-15 from UAM-Banco Santander FCH, MM and FGR also acknowledge CONICET (Project PIP 1035). XAFS experiments were partially supported by Project XAFS2-20150061 and SXS-20150180 (LNLS, Campinas, Brazil). The authors acknowledge Dr L. Giovanetti and Dr C. Huck-Iriart for SAXS experiments, which were performed at INIFTA, thanks to project "Nanopymes" (EropeAid/132184/ D/SUP/AR-Contract-896). DG acknowledges RYC-2012-09864, MAD2D-CM Program (S2013/MIT-3007) and SUPER-MNAN (ESP2015-65597-C4-3-R) for financial support.

\section{References}

1 C. d. M. Donega, Chem. Soc. Rev., 2011, 40, 1512.

2 A. Rogach, Semiconductor Nanocrystal Quantum Dots. Synthesis, Assembly, Spectroscopy and Applications, Springer-Verlag Wien, 2008.
3 M. V. Kovalenko, L. Manna, A. Cabot, Z. Hens, D. V. Talapin, C. R. Kagan, V. I. Klimov, A. L. Rogach, P. Reiss, D. J. Milliron, P. Guyot-Sionnnest, G. Konstantatos, W. J. Parak, T. Hyeon, B. A. Korgel, C. B. Murray and W. Heiss, ACS Nano, 2015, 9, 1012.

4 D. V. Talapin, J.-S. Lee, M. V. Kovalenko and E. V. Shevchenko, Chem. Rev., 2010, 110, 389.

5 C. B. Murray, D. J. Norris and M. G. Bawendi, J. Am. Chem. Soc., 1993, 115, 8706.

6 J. Tang, K. W. Kemp, S. Hoogland, K. S. Jeong, H. Liu, L. Levina, M. Furukawa, X. Wang, R. Debnath, D. Cha, K. W. Chou, A. Fischer, A. Amassian, J. B. Asbury and E. H. Sargent, Nat. Mater., 2011, 10, 765; Z. Ning, Y. Ren, S. Hoogland, O. Voznyy, L. Levina, P. Stadler, X. Lan, D. Zhitomirsky and E. H. Sargent, Adv. Mater., 2012, 24, 6295; A. H. Ip, S. M. Thon, S. Hoogland, O. Voznyy, D. Zhitomirsky, R. Debnath, L. Levina, L. R. Rollny, G. H. Carey, A. Fischer, K. W. Kemp, I. J. Kramer, Z. Ning, A. J. Labelle, K. W. Chou, A. Amassian and E. H. Sargent, Nat. Nanotechnol., 2012, 7, 577.

7 M. V. Kovalenko, M. Scheele and D. V. Talapin, Science, 2009, 324, 1417; M. V. Kovalenko, M. I. Bodnarchuk, J. Zaumseil, J.-S. Lee and D. V. Talapin, J. Am. Chem. Soc., 2010, 132, 10085; M. A. Boles and D. V. Talapin, Science, 2014, 344, 1340; F. Liu, J. Zhu, J. Wei, Y. Li, L. Hu, Y. Huang, O. Takuya, Q. Shen, T. Toyoda, B. Zhang, J. Yao and S. Dai, J. Phys. Chem. C, 2014, 118, 214; K. J. Schnitzenbaumer and G. Dukovic, J. Phys. Chem. C, 2014, 118, 28170; H. Zhang, J. Jang, W. Liu and D. V. Talapin, ACS Nano, 2014, 8, 7359; K. J. Schnitzenbaumer, T. Labrador and G. Dukovic, J. Phys. Chem. C, 2015, 119, 13314.

8 A. Nag, M. V. Kovalenko, J.-S. Lee, W. Liu, B. Spokoyny and D. V. Talapin, J. Am. Chem. Soc., 2011, 133, 10612.

9 H. Zhang, B. Hu, L. Sun, R. Hovden, F. W. Wise, D. A. Muller and R. D. Robinson, Nano Lett., 2011, 11, 5356.

10 M. L. Steigerwald, A. P. Alivisatos, J. M. Gibson, T. D. Harris, R. Kortan, A. J. Muller, A. M. Thayer, T. M. Duncan, D. C. Douglass and L. E. Brus, J. Am. Chem. Soc., 1988, 110, 3046.

11 J. Cizeron and M. P. Pileni, J. Phys. Chem., 1995, 99, 17410; C.-C. Chen, C.-Y. Chao and Z.-H. Lang, Chem. Mater., 2000, 12, 1516; N. Pinna, K. Weiss, H. Sack-Kongehl, W. Vogel, J. Urban and M. P. Pileni, Langmuir, 2001, 17, 7982; N. Pinna, K. Weiss, J. Urban and M. P. Pileni, Adv. Mater., 2001, 13, 261; Y.-W. Jun, S.-M. Lee, N.-J. Kang and J. Cheon, J. Am. Chem. Soc., 2001, 123, 5150; B. A. Simmons, S. Li, V. T. John, G. L. McPherson, A. Bose, W. Zhou and J. He, Nano Lett., 2002, 2, 263; S. Libert, D. V. Goia and E. Matijević, Langmuir, 2003, 19, 10673.

12 W. Xu and D. L. Akins, Mater. Lett., 2004, 58, 2623.

13 S.-Y. Chang, L. Liu and S. A. Asher, J. Am. Chem. Soc., 1994, 116, 6739.

14 R. Koole, M. M. van Schooneveld, J. Hilhorst, C. de Mello Donegá, D. C. Hart, A. van Blaaderen, D. Vanmaekelbergh and A. Meijerink, Chem. Mater., 2008, 20, 2503. 
15 L. Carbone, C. Nobile, M. De Giorgi, F. D. Sala, G. Morello, P. Pompa, M. Hytch, E. Snoeck, A. Fiore, I. R. Franchini, M. Nadasan, A. F. Silvestre, L. Chiodo, S. Kudera, R. Cingolani, R. Krahne and L. Manna, Nano Lett., 2007, 7, 2942.

16 W. W. Yu, L. Qu, W. Guo and X. Peng, Chem. Mater., 2003, 15, 2854; J. Jasieniak, L. Smith, J. v. Embden, P. Mulvaney and M. Califano, J. Phys. Chem. C, 2009, 113, 19468.

17 R. Xie, U. Kolb, J. Li, T. Basché and A. Mews, J. Am. Chem. Soc., 2005, 127, 7480; J. J. Li, Y. A. Wang, W. Guo, J. C. Keay, T. D. Mishima, M. B. Johnson and X. Peng, J. Am. Chem. Soc., 2003, 125, 12567; M. Ristov, G. J. Sinadinovski, I. Grozdanov and M. Mitreski, Thin Solid Films, 1989, 173, 53.

18 M. Grabolle, M. Spieles, V. Lesnyak, N. Gaponik, A. Eychmüller and U. Resch-Genger, Anal. Chem., 2009, 81, 6285.

19 F. Jalilehvand, Chem. Soc. Rev., 2006, 35, 1256.

20 B. Ravel and M. Newville, ATHENA, ARTEMIS, HEPHAESTUS: data analysis for X-ray absorption spectroscopy using IFEFFIT, 2005, 12, 537.

21 S. I. Zabinsky, J. J. Rehr, A. Ankudinov, R. C. Albers and M. J. Eller, Phys. Rev. B: Condens. Matter Mater. Phys., 1995, 52, 2995.

22 W.-Z. Li, L.-D. Wang, R. Gao, H.-P. Dong, G.-D. Niu, X.-D. Guo and Y. Qiu, Acta Phys. - Chim. Sin., 2013, 29, 2345.

23 R. M. Souto, S. González and A. Arévalo, J. Electroanal. Chem. Interfacial Electrochem., 1987, 216, 273; M. Krunks, J. Madarász, T. Leskelä, A. Mere, L. Niinistö and G. Pokol, J. Therm. Anal. Calorim., 2003, 72, 497; Q. Yu and C.-Y. Liu, J. Phys. Chem. C, 2009, 113, 12766; S. Jana, B. B. Srivastava and N. Pradhan, J. Phys. Chem. C, 2013, 117, 1183.

24 C. Schliehe, B. H. Juarez, M. Pelletier, S. Jander, D. Greshnykh, M. Nagel, A. Meyer, S. Foerster, A. Kornowski, C. Klinke and H. Weller, Science, 2010, 329, 550.
25 R. L. Penn, J. Phys. Chem. B, 2004, 108, 12707.

26 G. T. Patterson, Metals Speciation, Sepatation, and Recovery, CRC Press, 1990, vol. 2.

27 K. Žídek, M. Abdellah, K. Zheng and T. Pullerits, Sci. Rep., 2014, 4, 7244.

28 W. Lee, H. Kim, D.-R. Jung, J. Kim, C. Nahm, J. Lee, S. Kang, B. Lee and B. Park, Nanoscale Res. Lett., 2012, 7, 1 .

29 X. Zhong, M. Han, Z. Dong, T. J. White and W. Knoll, J. Am. Chem. Soc., 2003, 125, 8589.

30 J. R. McBride, A. D. Dukes, M. A. Schreuder and S. J. Rosenthal, Chem. Phys. Lett., 2010, 498, 1; M. A. Schreuder, J. R. McBride, A. D. Dukes, J. A. Sammons and S. J. Rosenthal, J. Phys. Chem. C, 2009, 113, 8169.

31 C. de Mello Donegá, M. Bode and A. Meijerink, Phys. Rev. B: Condens. Matter Mater. Phys., 2006, 74, 085320; M. Cirillo, T. Aubert, R. Gomes, R. Van Deun, P. Emplit, A. Biermann, H. Lange, C. Thomsen, E. Brainis and Z. Hens, Chem. Mater., 2014, 26, 1154; W. Nan, Y. Niu, H. Qin, F. Cui, Y. Yang, R. Lai, W. Lin and X. Peng, J. Am. Chem. Soc., 2012, 134, 19685.

32 F. Jalilehvand, Z. Amini and K. Parmar, Inorg. Chem., 2012, 51, 10619; M. Ranjbar, M. Yousefi, R. Nozari and S. Sheshmani, Int. J. Biomed. Nanosci. Nanotechnol., 2013, 9, 203.

33 R. Alonso Mori, E. Paris, G. Giuli, S. G. Eeckhout, M. Kavčič, M. Žitnik, K. Bučar, L. G. M. Pettersson and P. Glatzel, Anal. Chem., 2009, 81, 6516.

34 G. Van der Snickt, J. Dik, M. Cotte, K. Janssens, J. Jaroszewicz, W. De Nolf, J. Groenewegen and L. Van der Loeff, Anal. Chem., 2009, 81, 2600.

35 M. W. M. Y. M. Yiu, L. Liu, Y. Hu and T. K. Sham, AIP Conf. Proc., 2014, 1590, 26-31. 